\title{
TanDEM-X First DEM Acquisition: A Crossing Orbit Experiment
}

\author{
Paco López-Dekker, Member, IEEE, Pau Prats, Member, IEEE, Francesco De Zan, Daniel Schulze, \\ Gerhard Krieger, Senior Member, IEEE, and Alberto Moreira, Fellow, IEEE
}

\begin{abstract}
This letter describes the first interferometric acquisitions and results obtained by the TerraSAR-X add-on for Digital Elevation Measurements mission. Due to the large along-track separation between the two satellites during the approaching maneuver and the Earth's rotation, useful interferometric acquisitions were only possible at high latitudes. This resulted in a crossing angle between the ground tracks whose impact was corrected by acquiring the two synthetic-aperture radar images with an opposite squint. The still very large 2 -km cross-track baseline resulted in a 3.8-m interferometric height of ambiguity, producing extremely detailed images of the topography of the target area. Results acquired over the October Revolution Island, Russia, are shown and discussed.
\end{abstract}

Index Terms-Digital elevation models, radar interferometry, synthetic aperture radar.

\section{INTRODUCTION}

$\mathbf{O}$ N THE early morning of June 21, 2010, the TerraSAR-X (TSX) add-on for Digital Elevation Measurements (TDX) satellite, was successfully launched to initiate, in combination with its twin satellite TSX [1], the TDX mission [2]. On July 16 , just 25 days after the launch, the first highly experimental interferometric single-pass data were acquired, yielding the first digital elevation model (DEM) generated by the mission. This letter reports on these experiments and discusses these first interferometric results.

Throughout their expected 2.5 to 3 years of overlapping operating time, TSX and TDX will fly in close formation, forming a single-pass interferometric synthetic-aperture radar (InSAR) [3] with the goal of providing a global DEM with $12 \times 12 \mathrm{~m}^{2}$ horizontal sampling and $2-\mathrm{m}$ vertical relative accuracy. The nominal operating mode is a bistatic configuration in which either TSX or TDX transmits, and both satellites receive radar echoes.

For the monostatic commissioning phase of TDX, until October 2010, the two spacecraft were placed in similar orbits but with a large along-track separation. This included the monostatic commissioning phase, during which TSX and TDX where kept at a stable along-track distance of $20 \mathrm{~km}$. During this phase, the orbits were configured in order to compensate for the Earth's rotation, therefore producing almost identical ground tracks. This allowed repeat-pass InSAR measurements with a temporal lag of only a few seconds.

During the weeks previous to this first stable interferometric configuration, which is reached on July 20, TDX had to care-

Manuscript received September 22, 2010; revised January 16, 2011; accepted February 2, 2011

The authors are with the Microwaves and Radar Institute, German Aerospace Center, 82230 Oberpfaffenhofen, Germany (e-mail: francisco.dekker@ dlr.de).

Digital Object Identifier 10.1109/LGRS.2011.2127444 fully approach TSX from an initial along-track separation of $16000 \mathrm{~km}$. For two spacecraft placed in the same orbital plane, an along-track separation results in different ground tracks due to the Earth's rotation. The ground tracks have a maximum separation at the equator but cross at some point at high latitudes. As the tracks approach the crossing point, the interferometric cross-track baselines become smaller, but the tracks themselves become less parallel, which raises new issues. The InSAR under small crossing angles was studied and demonstrated in the context of Shuttle Imaging Radar-B (SIR-B) interferometric experiments [4].

Section II of this letter presents a reformulation of the crossing-orbit interferometry problem in terms of a 2-D spectral rotation of the sampled ground spectrum. Section III describes the experimental data acquisition, whereas the results are discussed in Section IV.

\section{THEORY}

Let us assume a SAR system that moves along the X-axis and, for simplification, over a flat terrain. The resulting SAR image samples the 2-D spectrum of the complex scattering coefficient $\sigma(x, y)$, so that the resulting image can be expressed, in the wavenumber domain, as

$$
S_{0}\left(k_{x}, k_{y}\right)=\sigma\left(k_{x}, k_{y}\right) W\left(k_{x}-k_{x 0}, k_{y}-k_{y 0}\right)
$$

where $W(\cdot, \cdot)$ is some baseband windowing function as follows:

$$
\begin{aligned}
& k_{x 0}=\frac{2 \pi f_{\mathrm{DC}_{0}}}{v_{x}}=2 k_{0} \sin \psi_{0} \sin \theta_{0} \\
& k_{y 0}=2 k_{0} \cos \psi_{0} \sin \theta_{0} .
\end{aligned}
$$

$y$ represents the ground range direction, $f_{\mathrm{DC}_{0}}$ is the Doppler centroid (DC), $v_{x}$ is the horizontal velocity of the system, $k_{0}$ is the wavenumber associated to the carrier frequency, $\psi_{0}$ is the ground projection of the squint angle, and $\theta_{0}$ is the incidence angle. Basically, this represents a rectangular spectrum centered at $\left(k_{x 0}, k_{y 0}\right)$. The nonzero $k_{y 0}$ term reveals that, for a squinted geometry, there is a projection of the flat Earth phase in the azimuth direction, indicating also a coupling between range and azimuth fringes.

Let us now consider a second image acquired by a system flying in a slightly different direction, which is rotated by angle $\alpha$, so that the coordinates in this rotated reference system are related to the coordinates in the original image by

$$
\begin{aligned}
& x=x^{\prime} \cos \alpha-y^{\prime} \sin \alpha \\
& y=x^{\prime} \sin \alpha+y^{\prime} \cos \alpha .
\end{aligned}
$$



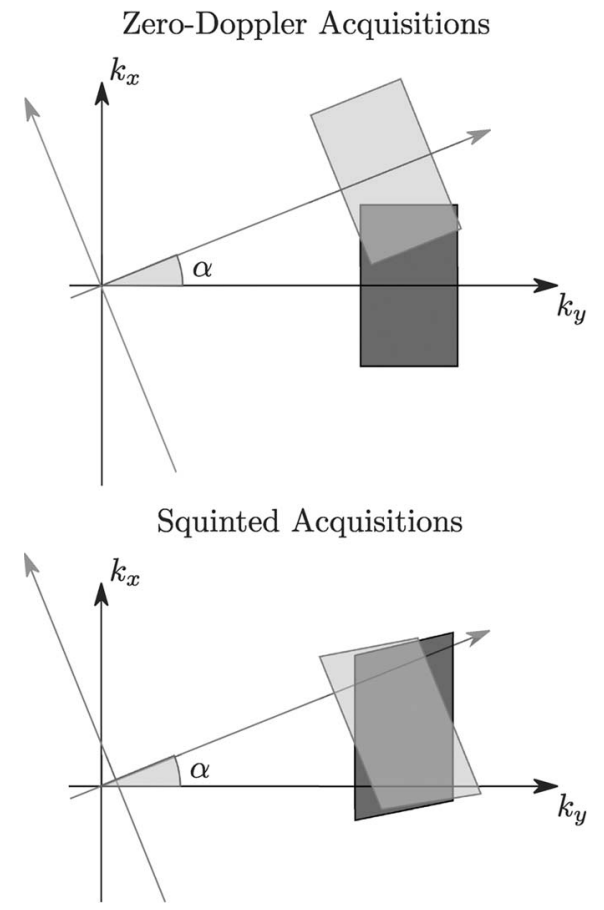

Fig. 1. Conceptual representation of the 2-D ground spectra for a pair of crossing acquisitions. Due to the rotation, if both images are acquired with $f_{\mathrm{DC}}=0$, the different parts of the 2-D ground spectrum are sampled. By acquiring the data with some relative squint, the spectral overlap is maximized.

In its own coordinate system, the spectrum of this second image will be

$$
S_{1}\left(k_{x}^{\prime}, k_{y}^{\prime}\right)=\sigma\left(k_{x}^{\prime}, k_{y}^{\prime}\right) W\left(k_{x}^{\prime}-k_{x 1}^{\prime}, k_{y}^{\prime}-k_{y 1}^{\prime}\right)
$$

which again will correspond to a rectangular spectrum centered this time at $\left(k_{x 1}^{\prime}, k_{y 1}^{\prime}\right)$. The same rotation applies also to the wavenumber domain as

$$
\begin{aligned}
& k_{x}=k_{x}^{\prime} \cos \alpha-k_{y}^{\prime} \sin \alpha \\
& k_{y}=k_{x}^{\prime} \sin \alpha+k_{y}^{\prime} \cos \alpha .
\end{aligned}
$$

In this way, in the original reference system, the 2-D spectrum of the second image is centered at

$$
\begin{aligned}
& k_{x 1}=k_{x 1}^{\prime} \cos \alpha-k_{y 1}^{\prime} \sin \alpha \\
& k_{y 1}=k_{x 1}^{\prime} \sin \alpha+k_{y 1}^{\prime} \cos \alpha
\end{aligned}
$$

and the entire spectrum can be written as

$$
S_{1}\left(k_{x}, k_{y}\right)=\sigma\left(k_{x}, k_{y}\right) W^{\prime}\left(k_{x}-k_{x 1}, k_{y}-k_{y 1}\right)
$$

where $W^{\prime}(\cdot, \cdot)$ represents the squinted spectral window. The two spectra are conceptually represented in the left diagram in Fig. 1 for the case in which both images are acquired with zero DC. In order to be able to form a useful interferogram, it is clear that the overlap between the spectra given by (1) and (2) should be maximized. It is now useful to define the following:

$$
\begin{aligned}
\Delta k_{x} & =k_{x 1}-k_{x 0} \\
\Delta k_{y} & =k_{y 1}-k_{y 0} .
\end{aligned}
$$

There will be spectral overlap if these two terms are smaller compared with $2 \pi / \delta x$ and $2 \pi / \delta y$, respectively, where $\delta y$ rep- resents the ground range resolution, and $\delta x$ represents the azimuth resolution. For small values of $\alpha$, the similar incidence angles $\Delta \theta=\theta_{1}-\theta_{0} \approx 0$, and the small squint angle difference $\left.\Delta \Psi=\Psi_{1}-\Psi_{0}\right)$; these spectral shifts reduce to

$$
\begin{aligned}
& \Delta k_{x} \approx 2 k_{0}\left(\sin \psi_{0} \cos \theta_{0} \Delta \theta+\cos \psi_{0} \sin \theta_{0}(\Delta \psi-\alpha)\right) \\
& \Delta k_{y} \approx 2 k_{0}\left(\cos \psi_{0} \cos \theta_{0} \Delta \theta-\sin \psi_{0} \sin \theta_{0}(\Delta \psi-\alpha)\right) .
\end{aligned}
$$

Assuming also small squints (or DCs) yields

$$
\begin{aligned}
& \Delta k_{x} \approx 2 k_{0} \sin \theta_{0}(\Delta \psi-\alpha)=\frac{2 \pi \Delta f_{\mathrm{DC}}}{v_{x}}-2 k_{0} \alpha \sin \theta_{0} \\
& \Delta k_{y} \approx 2 k_{0} \cos \theta_{0} \Delta \theta
\end{aligned}
$$

The range spectral shift $\Delta k_{y}$ reduces to the typical spectral shift studied for parallel orbits [5]. Note that a variation of the incidence angle given by $\Delta \theta$ is the result of a normal baseline component. The azimuth spectral shift $\Delta k_{x}$ depends on the angle between the orbits and on the relative squint between the two acquisitions. It is zero if the horizontal rotation of the reference system $\alpha$ is equal to the ground projection of the squint angle $\Delta \psi$. Geometrically, this means that the target is being observed from the same ground-projected direction.

The alignment of the spectra after applying a relative squint is illustrated by the right diagram in Fig. 1, where all the angles are clearly exaggerated, and a zero cross-track baseline is assumed. In practice, for small rotations and squint angles and considering that $k_{y 0} \gg 2 \pi / \delta_{y}$, their effect is practically reduced to a spectral shift in azimuth.

A pair of images acquired with different squints in order to compensate the crossing angle will present different Doppler spectra in their respective range-azimuth coordinates. It is interesting to note, however, that the spectral components will be automatically aligned by interferometric processing, i.e., the flat earth phase removal. Introducing a relative squint and, hence, a $f_{\mathrm{DC}}$ offset, to compensate the azimuth spectral shift is thus equivalent to applying a center frequency offset in order to compensate a large range spectral shift.

Fig. 2 shows the spectral shifts due to the crossing orbit and the cross-track baseline for a TDX-like system and an orbit, assuming that the two spacecraft are in an identical orbit (in an intertial frame) but with an along-track separation of $500 \mathrm{~km}$. The spectral shifts, which correspond to an incidence angle of $40^{\circ}$, are represented as a function of the latitude of the imaged area. As expected, at the equator, the range spectral shift is maximized, the azimuth spectral shift is zero due to the parallel tracks, and the situation is reversed at large latitudes. For most latitudes, the range spectral shift is larger than the critical one given by $2 \pi / \delta y$, which is represented in the figures by dashed and dotted lines for a pulse bandwidth of 150 and $300 \mathrm{MHz}$, respectively. The lower plot represents the differential DC required to compensate the spectral shift in azimuth. In practice, considering nonidentical orbits results in an extra baseline component and, therefore, an extra range spectral shift term.

\section{EXPERIMENT DESCRIPTION}

Two pairs of acquisitions were programmed: an ascending pass over Greenland on July 15 at 21:27 coordinated universal 

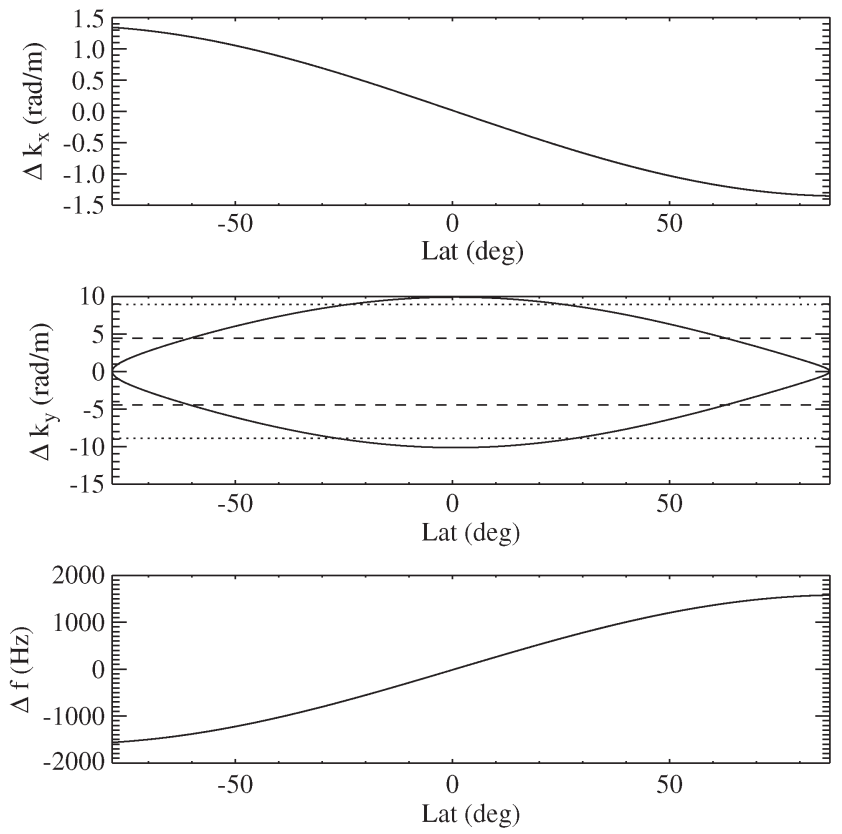

Fig. 2. Theoretical spectral shifts in (top) azimuth and (center) range and (bottom) the DC offset required to align the azimuth spectra as a function of latitude assuming identical orbits with a 500-km along-track separation and an incidence angle of $45^{\circ}$. The dashed and dotted lines show the critical baselines for a pulse bandwidth of 150 and $300 \mathrm{MHz}$, respectively.

TABLE I

Crossing Orbit InSAR Parameters at $80^{\circ}$ Latitude

\begin{tabular}{|c|c|c|c|}
\hline & Predicted & Configured & Real \\
\hline$\Delta k_{x}(\mathrm{rad} / \mathrm{m})$ & -0.92 & - & -0.93 \\
$\Delta k_{y}(\mathrm{rad} / \mathrm{m})$ & -0.81 & - & -0.825 \\
$\Delta f_{D C}(\mathrm{~Hz})$ & 1075 & 1240 & 1093 \\
Relative squint & $0.126^{\circ}$ & $0.145^{\circ}$ & $0.127^{\circ}$ \\
\hline
\end{tabular}

time (UTC) and a descending one over the October Revolution Island in the Russian Arctic, on July 16 at 00:41 UTC. The results presented in this letter correspond to the start of this second acquisition. Based on the predicted TSX and TDX orbits, the expected azimuth spectral shift was $-0.92 \mathrm{rad} / \mathrm{m}$ at a $40^{\circ}$ off-nadir look angle, requiring a DC offset of $1.1 \mathrm{kHz}$, which is a significant fraction of the Doppler bandwidth. One possibility, in line with the SIR-B experiment reported in [4], would have been to acquire both images with the nominal zero DC and keep the reduced common band during the interferometric processing. However, for these experiments, it was decided to exploit the flexibility of TSX and TDX active antenna arrays and steer the beams in azimuth in order to obtain the desired DC offset. Desired $\Delta f_{\mathrm{DC}}$ corresponds to a relative azimuth antenna squint of $0.13^{\circ}$, with the trailing TDX system looking forward with respect to TSX. In practice, TSX was configured with a backward squint of $-0.066^{\circ}$ and TDX with a forward squint of $0.079^{\circ}$, resulting in a relative squint of $0.145^{\circ}$. As shown in Table I, the spectral shifts and the required squint calculated with the precise orbits available after the acquisitions matched almost perfectly the predicted ones.

The cross-track baseline varied during the 29-s acquisition (i.e., 200-km strip image) from 1.9 to $2.4 \mathrm{~km}$. For the results presented in the following, it was approximately $2 \mathrm{~km}$, which corresponds to a height of ambiguity of $3.8 \mathrm{~m}$. This is about an order of magnitude smaller than the nominal values assumed for the mission (in bistatic operations). With this small height of ambiguity, for relatively flat areas, the expected relative height accuracy, excluding systematic errors, is in the order of $10 \mathrm{~cm}$. It must be pointed out, however, that this performance is lost as soon as the topography causes phase unwrapping errors or due to strong topography-induced geometric decorrelation. The along-track separation of the satellites was approximately $380 \mathrm{~km}$, so that the two images were acquired with a time separation of $48 \mathrm{~s}$.

\section{RESUlts}

The results shown in Fig. 3 correspond to a $20-\mathrm{km}$ ground range by $31 \mathrm{~km}$ in the azimuth strip of the October Revolution Island. The slant-range-azimuth images show, from left to right, the relative brightness, the interferometric phase, and interferometric coherence. The images have been rotated, so that north is roughly at the top. A $9 \times 6$ multilook window has been applied to the data yielding $12 \times 12 \mathrm{~m}^{2}$ pixels. SAR focusing and interferometric processing were performed using the Microwaves and Radar Institute's experimental interferometric processor [6]. The data were processed without any azimuth common-band spectral filtering. In range, the spectral shift was in the order of $20 \%$ of the available bandwidth, making common-band filtering a necessity. The fine coregistration step was performed using a DEM generated with a later 200-m-baseline TDX acquisition. This DEM was also used to flatten the interferometric phase, thereby simplifying the unwrapping of the residual phase and greatly improving the estimation of the coherence. The high interferometric coherence obtained, up to 0.95 in some areas, is a good illustration of the potential of the mission.

Most of the scene corresponds to sea ice, which can be identified by low reflectivity (associated to melting ice, as expected for this time of the year) and the almost constant residual interferometric phase. The diagonal linear-phase component visible in this is probably due to a residual geometric error. Some small very low reflectivity and low-coherence features in the ice correspond probably to pools of melted water. The high-reflectivity regions with clear topography-related fringes correspond to, probably ice covered, land. The low-reflectivity region with well-organized fringes at the left side of the images is an outflow glacier. It is worth emphasizing the small $3.8-\mathrm{m}$ height of ambiguity, meaning that the high-frequency interferometric fringes correspond in reality to smooth and gentle slopes.

Fig. 4 shows a zoom-in view around a particularly interesting feature in the images. The amplitude and coherence suggest a series of connected or almost connected water pools and some apparent cracking line in the ice. Approximately at the middle of the image, there is an area with nearly parallel and almost perfect azimuth fringes. This type of an interferometric signature has been identified by several authors [7], [8] as the result of a rotation along a vertical axis occurred during the interval betwen the acquisitions. Interestingly enough, this phenomenon, which results in an apparent spectral shift in azimuth, is exactly equivalent to having nonparallel orbits. Since from the point of view of SAR image formation, the orbit of interest is that with respect to the target; there is no difference 


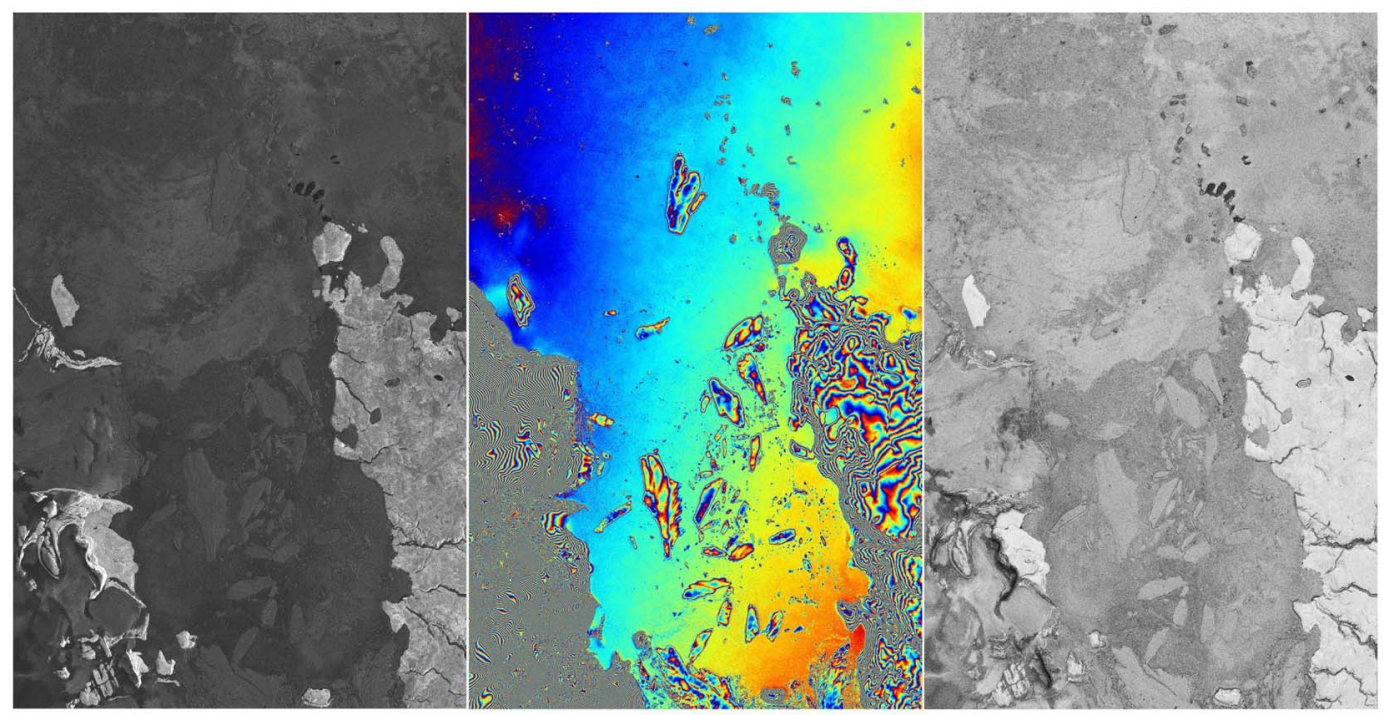

Fig. 3. (From left to right) Relative brightness, interferometric phase, and interferometric coherence of an area corresponding to the northeastern coast of the October Revolution Island in the Russian Arctic. The images are in slant-range (from right to left)-azimuth (top to bottom) coordinates.
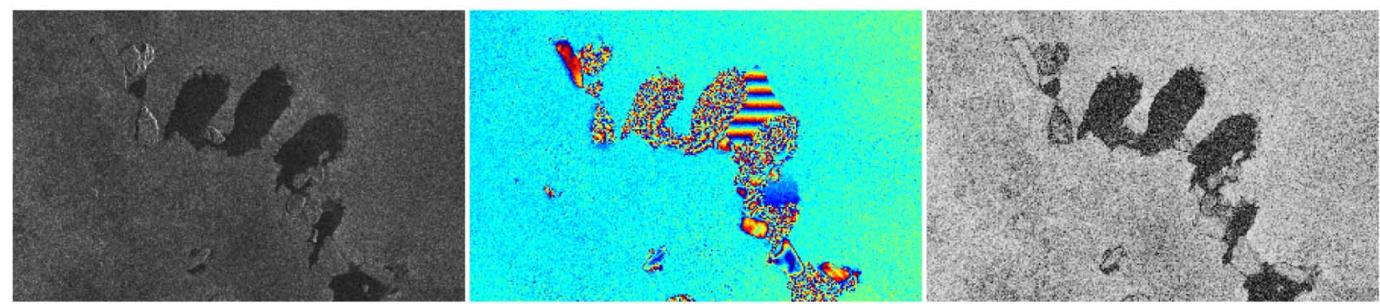

Fig. 4. These zoom-out images of reflectivity, the interferometric phase, and coherence show a closer look at what look like pools of melted ice. Lines in the amplitude and coherence images suggest cracks in the ice sheets. Close to the center of the detail, a region shows with nearly perfect parallel fringes that correspond to a linear phase in azimuth, which can be explained by a rotation along a vertical axis of the area.

between rotating the orbit and rotating the target. The actual fringe rate depends on the rotation angle $\alpha_{r}$ of the target area and the incidence angle and can be derived directly from (10) as follows:

$$
f_{a z}=2 k_{0} \alpha_{r} \sin \theta \quad[\mathrm{rad} / \mathrm{m}] .
$$

Normalizing to the azimuth pixel spacing $\delta x$, for an azimuth pixel spacing of $1.8 \mathrm{~m}$, a wavelength of $3 \mathrm{~cm}$, and an incidence angle of $45^{\circ}$, a rotation of just $0.7^{\circ}$ would cause a frequency of $1 \mathrm{~Hz} / \mathrm{sample}$, thus causing total decorrelation. The azimuth fringes in Fig. 4 correspond to a rotation in the order of $0.011^{\circ}$.

This particular example strongly shows that with a 48-s delay, the along-track interferometric phase component (or differential InSAR component) cannot be ignored. This raises the question of to which extent the measured residual phases in other areas have an along-track component and illustrates the importance of the close formation configuration planned for most of the mission.

Fig. 5 shows a rendering of the DEM obtained, with height ranging from $0 \mathrm{~m}$ at sea level to $585 \mathrm{~m}$ at the southwestern corner of the image. To better illustrate the DEM quality, the top panel in Fig. 6 shows a profile of the DEM along the red line in Fig. 5. The profile shows blocks of sea ice rising several meters above the frozen sea-level, and land raising several tens of meters. The center panel in the figure shows

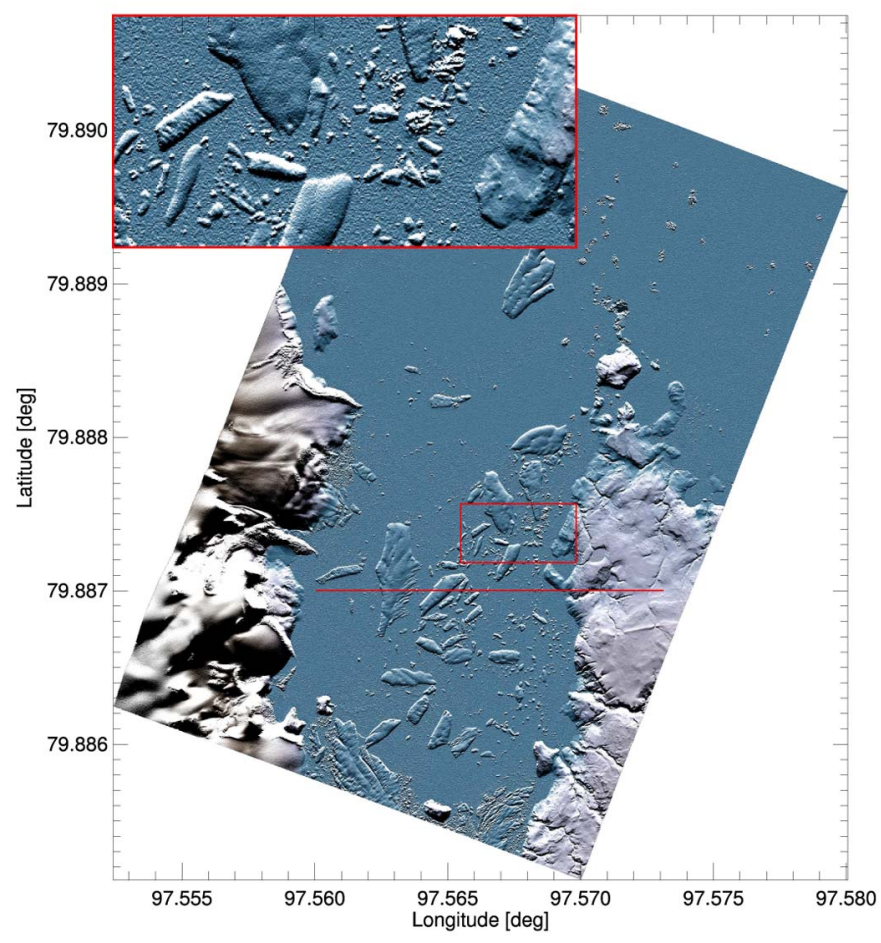

Fig. 5. Geocoded and rendered DEMs. The zoom-in image (red rectangle) illustrates the high level of topographic detail. 

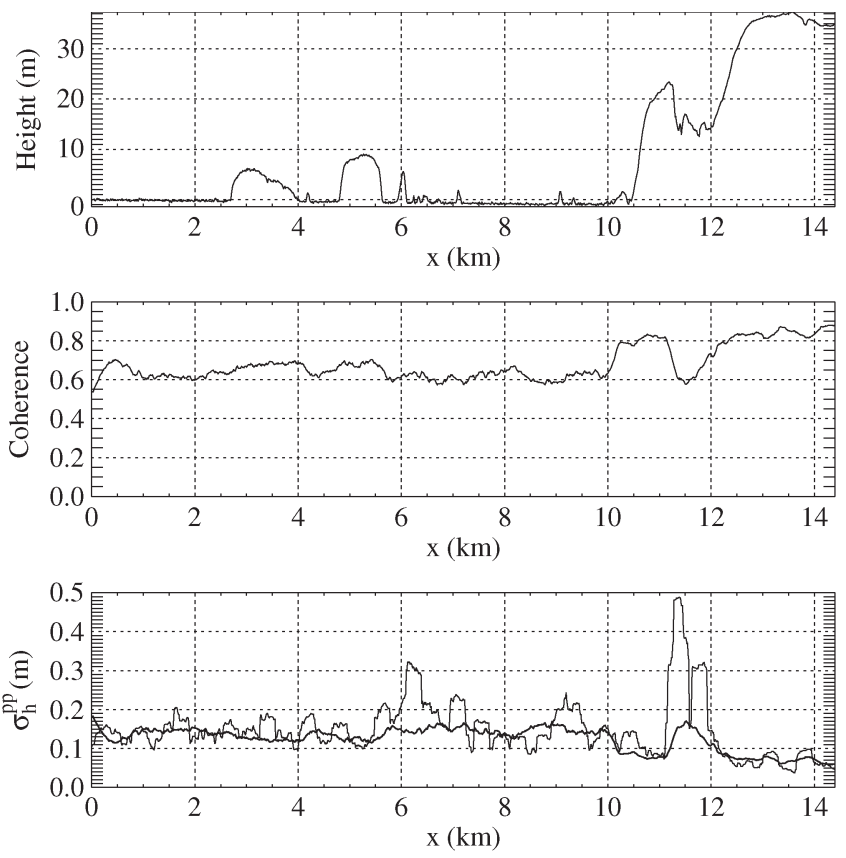

Fig. 6. (From top to bottom) the DEM profile, coherence, and the estimated standard deviation of the point-to-point height errors estimated from the coherence (thick black line) and from the high-frequency component of the DEM profile. The profiles correspond to the West to East line in Fig. 5.

the estimated coherence, which varies between 0.6 over the frozen sea to around 0.9 over land. The bottom panel shows the estimated standard deviation of the point-to-point relative height error $\sigma_{h}^{\mathrm{pp}}$, not including systematic errors. The heavy black line shows the error derived from the coherence and the effective number of looks (approximately 30 in this case) following the derivation in [2]. The gray line shows the sliding variance of the high-pass filtered DEM profile. Since highpass filtering also eliminated the low-frequency component of the DEM error, this standard deviation was corrected to compensate this missing noise power, implicitly assuming that the DEM error can be characterized as white noise. It was also multiplied by a $\sqrt{2}$ factor to represent a point-to-point error. This second estimate overestimates the error in regions with high-frequency topographic variations. The standard deviation of the estimated point-to-point errors is, in most cases, in the $10-20 \mathrm{~cm}$ range, with values down to $5-6 \mathrm{~cm}$ in the highcoherence areas.

\section{Discussion AND CONCLUSION}

This letter has presented and discussed the first interferometric acquisition of the TDX mission. It has been shown how the crossing angle between the orbits introduces a spectral shift in the azimuth direction, which can be compensated by applying an appropriate squint during the acquisitions. While the problem itself is not new, this represents the first demonstration of an active compensation, which extends the possibility of InSAR acquisitions under crossing orbits to crossing angles significantly larger than the antenna beamwidth. This technique may be of interest to future missions.

This experiment has also illustrated experimentally the level of topographic detail that can be obtained using TSX-class satellites, where the high spatial resolution allows for relatively very large baselines. The resulting unique datasets should be further analyzed to explore their full potential. This should lead to the design of future experiments using TSX, TDX or similar systems and help the definition of future missions [9].

\section{REFERENCES}

[1] R. Werninghaus and S. Buckreuss, "The TerraSAR-X mission and system design," IEEE Trans. Geosci. Remote Sens., vol. 48, no. 2, pp. 606-614, Feb. 2010.

[2] G. Krieger, A. Moreira, H. Fiedler, I. Hajnsek, M. Werner, M. Younis, and M. Zink, "TanDEM-X: A satellite formation for high-resolution SAR interferometry," IEEE Trans. Geosci. Remote Sens., vol. 45, no. 11, pp. 3317-3341, Nov. 2007.

[3] L. Graham, "Synthetic interferometer radar for topographic mapping," Proc. IEEE, vol. 62, no. 6, pp. 763-768, Jun. 1974.

[4] A. K. Gabriel and R. M. Goldstein (1988, May). Crossed orbit interferometry: Theory and experimental results from SIR-B. Int. J. Remote Sens. [Online]. 9(5), pp. 857-872. Available: http://www.informaworld.com/ $10.1080 / 01431168808954901$

[5] F. Gatelli, A. Monti Guarnieri, F. Parizzi, P. Pasquali, C. Prati, and F. Rocca, "The wavenumber shift in SAR interferometry," IEEE Trans. Geosci. Remote Sens., vol. 32, no. 4, pp. 855-865, Jul. 1994.

[6] P. Prats, M. Rodriguez-Cassola, L. Marotti, M. Nannini, G. Krieger, and A. Reigber, "TAXI: A versatile processing chain for experimental TanDEM-X product evaluation," in Proc. Int. Geosci. Remote Sens. Symp., Honolulu, HI, 2010, pp. 4059-4062.

[7] G. Peltzer, K. W. Hudnut, and K. L. Feigl, "Analysis of coseismic surface displacement gradients using radar interferometry: New insights into the Landers earthquake," J. Geophys. Res., vol. 99, no. B11, pp. 21 971-21 981, 1994.

[8] E. Rignot and D. R. MacAyeal, "Ice-shelf dynamics near the front of the Filchner-Ronne ice shelf, Antarctica, revealed by SAR interferometry," J. Glaciol., vol. 44, no. 147, pp. 405-418, 1998.

[9] T. Börner, F. De Zan, F. López-Dekker, G. Krieger, I. Hajnsek, K. Papathanassiou, M. Villano, M. Younis, A. Danklmayer, W. Dierking, T. Nagler, H. Rott, S. Lehner, T. Fügen, and A. Moreira, "SIGNAL: SAR for ice, glacier and global dynamics," in Proc. IEEE IGARSS, Honolulu, HI, Jul. 2010, pp. 2884-2887. 